Research Article

\title{
Comparison of Chemical Constituents in Pseudostellariae Radix with Different Dosage Forms Based on HPLC-Q-Exactive Orbitrap/MS Combined with Multivariate Statistical Analysis
}

\author{
Yujiao Hua $(\mathbb{D}$, Xiaoyuan Liu $(\mathbb{D}$, and Fen Xie \\ Department of Clinical Pharmacy, Affiliated Hospital of Jiangnan University, Wuxi 214000, China \\ Correspondence should be addressed to Fen Xie; 303991002@qq.com
}

Received 15 December 2020; Revised 6 April 2021; Accepted 27 April 2021; Published 10 May 2021

Academic Editor: Amir Syahir

Copyright (c) 2021 Yujiao Hua et al. This is an open access article distributed under the Creative Commons Attribution License, which permits unrestricted use, distribution, and reproduction in any medium, provided the original work is properly cited.

\begin{abstract}
Background. Pseudostellariae Radix (PR) is an important traditional Chinese herbal medicine with vast clinical consumptions, which has two different dosage forms, PR decoction pieces and PR formula granules. However, these two forms are bound to have an impact on the accumulation of the effective components in PR, so the effectiveness of clinical use cannot be guaranteed. Objective. To determine the effective composition of PR. Methods. In this research, PR decoction pieces and formula granules were collected, and their composition was detected by HPLC-Q-Exactive Orbitrap/MS; multivariate statistical analysis was used to distinguish differential metabolites between PR decoction pieces and formula granules. Results. A clear cut difference in the composition of the two groups was observed. 98 differential chemical constituents could be identified in the positive mode, while 52 differential chemical compositions could be identified in the negative mode. The differential chemical compositions were mainly concentrated in flavonoids, organic acids, fatty acids, and amino acids and present different change rules, mainly involved in the isoquinoline alkaloid biosynthesis metabolic pathways. Conclusions. This study provides basic information to reveal the influence law of different dosage forms on the metabolite synthesis and quality formation mechanism of PR.
\end{abstract}

\section{Introduction}

Pseudostellariae Radix (PR) is an arid tuberous root of P. heterophylla (Miq.) Pax ex Pax et Hoffm [1]. It is a kind of important traditional Chinese herbal medicine which has the functions of promoting immunity, relieving cough, antitumor, antioxidation, and protecting myocardial function [2]. This herbal medicine is used wildly for its positive effects. It is reported that PR can be used for inappetence [3], thirst [4], debility [5], diabetes [6], and weakness after illness [7]. Currently, PR decoction pieces and formula granules are utilized in a Chinese hospital; among them, PR formula granules is a novel form of decoction pieces, which is prepared by water extraction, concentration, drying, and granulation of the PR decoction pieces $[8,9]$. These two types of PR used have their own advantages and disadvantages. One of the advantages of PR decoction pieces being that it has been prescribed since thousands of years as a potent drug with high traditional following. On the other hand, the advantages of PR formula granules lie in small package, multiple varieties, and multiple specifications, and the relevant parameters of drugs are clearly marked, which is conducive to the identification and selection of medicinal materials by patients; also, the formula granules are easy for patients to take home $[10,11]$.

The different dosage forms are bound to have an impact on the accumulation of its effective constituents, so the effectiveness of clinical use cannot be guaranteed, and the active components of these two dosage forms have not been studied in depth. In order to have a more comprehensive understanding on the synthesis and accumulation of metabolites in PR with different dosage forms, as well as the difference in quality between PR decoction pieces and formula granules, it is necessary to build a quality analysis method for the overall chemical constituents of PR with different dosage forms. 
Metabolomics technology is a new omics technology developed in the mid-1990s, among which plant metabolomics is a high-throughput, unbiased, and comprehensive analysis technology for metabolomics in plant extracts, and it is especially fit for the analysis of multicomponent complex systems of TCM [12-14]. In recent years, liquidmass coupling technology is widely used in the qualitative and quantitative research of complex TCM systems $[15,16]$, and the commonly used mass spectrometry techniques include quadrupole mass spectrometry, ion trap mass spectrometry, time-of-flight mass spectrometry, and compound tandem mass spectrometry [17-20]. The structure of the compounds can be rapidly identified based on the fragmentation information of the multistage mass spectrometry compounds and related database. Liquid phase with high efficiency and rapid separation performance combined with sensitive and accurate mass spectrometry is one of the most promising metabolomics technologies and has been widely used in the analysis of polar compounds, thermal unstable compounds, and macromolecular compounds.

In this study, an accurate and reliable method based on HPLC-Q-Exactive Orbitrap/MS coupled with multivariate statistical analysis and signal pathway analysis has been developed to analyze the differential chemical compositions and major metabolic pathways in PR decoction pieces and formula granules, exploring the dynamic change law of different dosage forms of PR. It is expected to provide basic data for revealing the influence of different dosage forms on the synthesis and accumulation of metabolites of PR and for discussing the formation mechanism of the quality in PR decoction pieces and formula granules.

\section{Materials and Methods}

2.1. Chemicals and Reagents. Formic acid and acetonitrile of HPLC grade were purchased from Sigma-Aldrich (St. Louis, MO, USA) and Merck (Darmstadt, Germany), respectively. Ultrapure water was prepared using a Milli-Q purifying system (Millipore, Bedford, MA, USA) under a resistivity of $18.2 \mathrm{M} \Omega / \mathrm{cm}$, and other reagent solutions such as methanol and 2-chloro-L-phenylalanine were analytical grade (Merck, Darmstadt, Germany).

2.2. Plant Materials. Five PR decoction pieces were purchased from Suzhou Tianling Chinese Herbal Medicine Co. Ltd., Jiangsu Province, China. Five PR formula granules were purchased from Jiangyin Tianjiang Pharmaceutical Co. Ltd., Jiangsu Province, China. The botanical origin of the materials was identified by Professor Xunhong Liu (Department for Authentication of Chinese Medicines, Nanjing University of Chinese Medicine, China), and the voucher specimens were deposited at TCM pharmacy of Affiliated Hospital of Jiangnan University.

2.3. Sample Preparations. PR decoction pieces and PR formula granules were naturally put at room temperature prior to HPLC-Q-Exactive Orbitrap/MS analysis. The dried
PR decoction pieces and formula granules were pulverized into homogeneous powders (80 mesh). Powder samples $(0.1 \mathrm{~g})$ were accurately weighed out and transferred to a $25 \mathrm{~mL}$ conical flask equipped with a stopper. Then, samples were applied to extraction procedure, extracted with $800 \mu \mathrm{L}$ of methanol and $10 \mu \mathrm{L}$ of internal standard $(2.8 \mathrm{mg} / \mathrm{mL}$, DLo-chlorophenylalanine). All samples were grinded to fine powder using a grinding mill at $65 \mathrm{~Hz}$ for $90 \mathrm{~s}$, and then, they were ultrasonicated for $30 \mathrm{~min}$, by $40 \mathrm{KHz}$ and let stand for 1 hour at $-20^{\circ} \mathrm{C}$. The samples were centrifuged at $12000 \mathrm{rpm}$ and $4^{\circ} \mathrm{C}$ for $15 \mathrm{~min}$, and the supernatants were filtered through a $0.22 \mu \mathrm{m}$ membrane before injection into the HPLC system.

2.4. HPLC-Q-Exactive Orbitrap/MS Conditions. The HPLC analysis was performed on the Ultimate 3000 HPLC system (Thermo, Waltham, MA, USA). A hyper gold C18 column $(100 \mathrm{~mm} \times 2.1 \mathrm{~mm} \times 1.9 \mu \mathrm{m})$ (Thermo, Waltham, MA, USA) was used to carry out the chromatographic separation at $40^{\circ} \mathrm{C}$. The mobile phase consisted of eluent $\mathrm{A}$ (water $+5 \%$ acetonitrile $+0.1 \%$ formic acid, $v / v$ ) and eluent B (acetonitrile $+0.1 \%$ formic acid, $v / v$ ) with a flow rate of $0.35 \mathrm{~mL} / \mathrm{min}$ with a liner gradient program: $100 \%-80 \% \mathrm{~A}$ from 0 to $1.5 \mathrm{~min}, 80 \%-0 \% \mathrm{~A}$ from 1.5 to $9.5 \mathrm{~min}, 0 \% \mathrm{~A}$ from 9.5 to $14.5 \mathrm{~min}, 0 \%-100 \% \mathrm{~A}$ from 14.5 to $14.6 \mathrm{~min}$, and $100 \%$ A from 14.6 to $18 \mathrm{~min}$.

Mass spectrometry detection was performed on Q-Exactive Orbitrap/MS (Thermo, Waltham, MA, USA) equipped with an electrospray ionization (ESI) source operating in the positive and negative ion modes. The scan range was $\mathrm{m} / \mathrm{z} 50-1000$ and $\mathrm{m} / \mathrm{z} 50-1100$ in positive and negative ion modes, respectively. The optimized instrumental parameters were as follows: heater temperature was $300^{\circ} \mathrm{C}$, sheath gas flow rate was $45 \mathrm{arb}$, Aux gas flow rate was 15 arb, sweep gas flow rate was 1 arb, and capillary temperature was $350^{\circ} \mathrm{C}$. The spray voltage was floating at $3.0 \mathrm{KV}$ $\left(\mathrm{ESI}^{+}\right)$or $3.2 \mathrm{KV}\left(\mathrm{ESI}^{-}\right)$. S-lens RF level was $30 \%\left(\mathrm{ESI}^{+}\right)$or $60 \%\left(\mathrm{ESI}^{-}\right)$.

2.5. Data Analysis. The data were performed feature extraction and preprocessed with Compound Discoverer software (Thermo, Waltham, MA, USA) and then normalized and edited into a two-dimensional data matrix by Excel 2010 software (Redmond, WA, USA), including retention time (RT), compound molecular weight (compMW), observations (samples), and peak intensity. The edited data matrix was imported into SIMCA-P 13.0 software (Umetrics, Umea, Sweden) for multivariate statistical analysis. Principal component analysis (PCA) was performed to intuitively express the difference in chemical compositions between PR decoction pieces and PR formula granules by observing the aggregation of each sample. Partial least squares discriminant analysis (PLS-DA) and orthogonal least squares discriminant analysis (OPLS-DA) were performed to further classify the samples. Two parameters, $R^{2} Y$ and $Q^{2}$, were used to evaluate the model, the closer $R^{2} Y$ is to 1 , the more stable the model is, and $Q^{2}>0.5$ indicates a high prediction rate. Variable importance in the projection (VIP) $>1$ via OPLS-DA analysis 


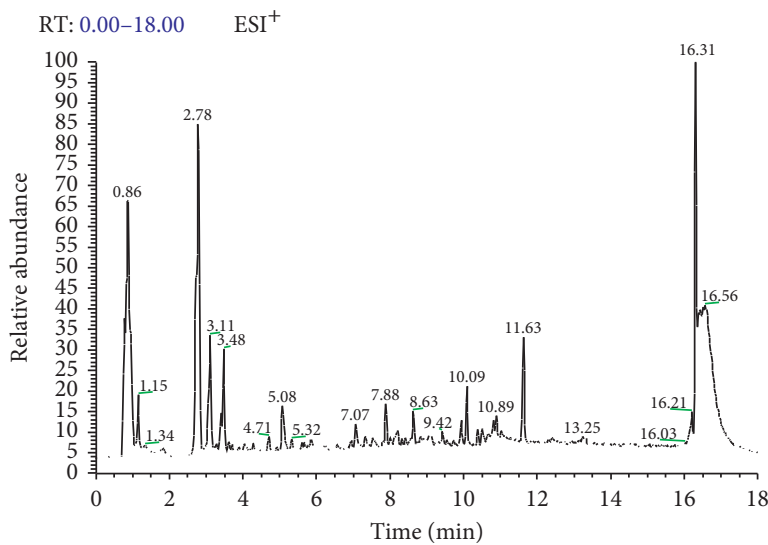

(a)

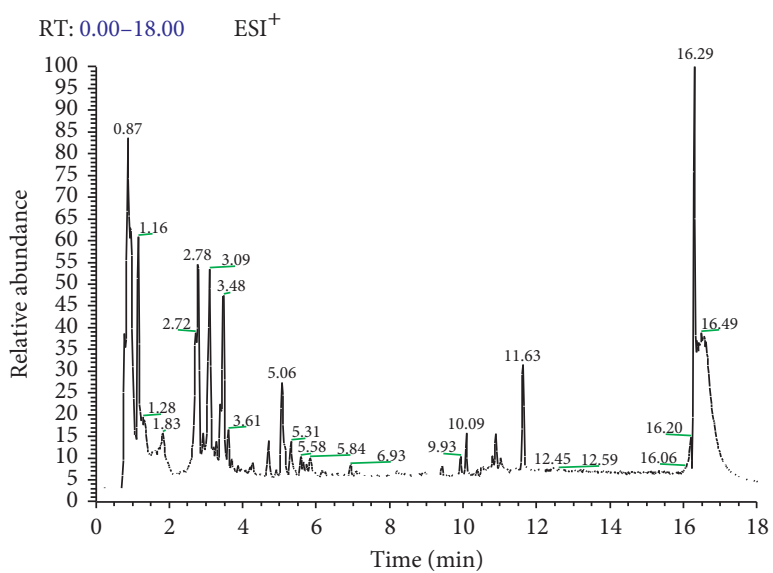

(c)

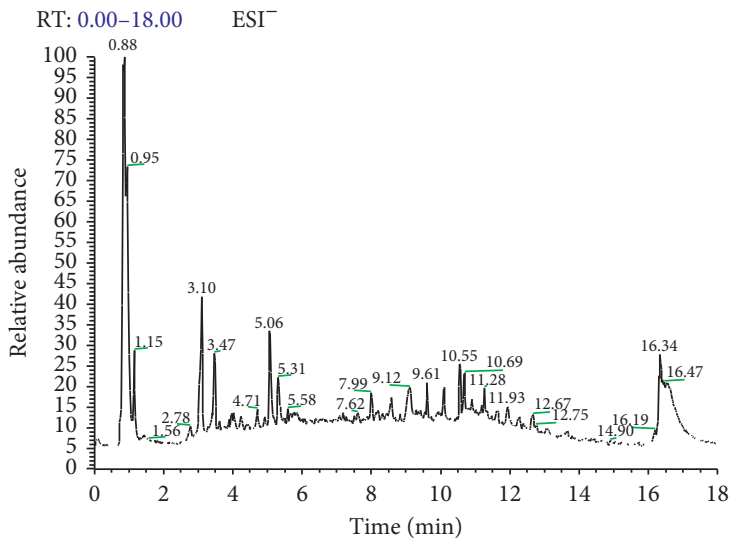

(b)

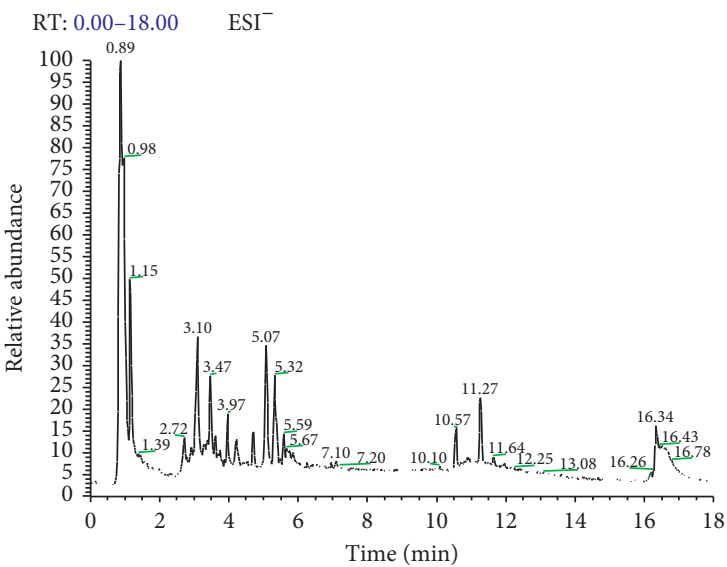

(d)

Figure 1: BPC of PR decoction pieces (a-b) and PR formula granules (c-d) in both positive and negative modes.

and the $p$ value of the $t$-test $(p<0.05)$ were used to find potential metabolites that significantly contributed to the difference among the groups [21]. Biochemical databases, METLIN (http://metlin.scripps.edu/), HMDB (http://www. hmdb.ca/), KEGG (http://www.kegg.com/), and MetaboAnalyst (http://www.metaboanalyst.ca/), were used to identify potential metabolites. According to the data of MetaboAnalyst, the impact value threshold was set at 0.6 , and therefore, the most important potential metabolic pathways were filtered out [22].

\section{Results and Discussion}

3.1. Based Peak Chromatogram. The extraction conditions, including extraction (ultrasonic and refluxing extraction), extraction solvent (100\% methanol, $70 \%$ methanol, $50 \%$ methanol, and $30 \%$ methanol), and extraction time $(15,30$, 45,60 , and $75 \mathrm{~min}$ ) were optimized to acquire the most outstanding extraction efficiency. The results showed that the chromatographic peak shape and relative peak area were superior to the others under ultrasonic extraction with $100 \%$ methanol for $30 \mathrm{~min}$.

In order to gain fast effective analysis, a hyper gold C18 column $(100 \mathrm{~mm} \times 2.1 \mathrm{~mm} \times 1.9 \mu \mathrm{m})$ column was employed for this experiment. Different mobile phases (including methanol-water, acetonitrile-water, methanol- $0.1 \%$ formic acid water, acetonitrile- $0.1 \%$ formic acid water, acetonitrile with $0.1 \%$ formic acid water-acetonitrile with $0.1 \%$ formic acid, and $5 \%$ acetonitrile with $0.1 \%$ formic acid water-acetonitrile with $0.1 \%$ formic acid), flow rate $(0.25,0.35$, and $0.45 \mathrm{~mL} / \mathrm{min})$, and column temperature $\left(35,40\right.$, and $\left.45^{\circ} \mathrm{C}\right)$ were examined and compared. As a result, a 5\% acetonitrile with $0.1 \%$ formic acid water-acetonitrile with $0.1 \%$ formic acid at a flow rate of $0.35 \mathrm{~mL} / \mathrm{min}$ and a column temperature of $40^{\circ} \mathrm{C}$ was found satisfactory for separation of molecules in a short time.

According to the set conditions of sample treatment, liquid chromatography and mass spectrometry were determined. The based peak chromatogram (BPC) of PR decoction pieces and formula granules obtained from the analysis in both positive and negative modes is shown in Figure 1.

\subsection{Multivariate Statistical Analysis}

3.2.1. Principal Component Analysis. Based on LC-MS spectra, chemical classification of all samples was carried out by multivariate data analysis, which aims to evaluate the 


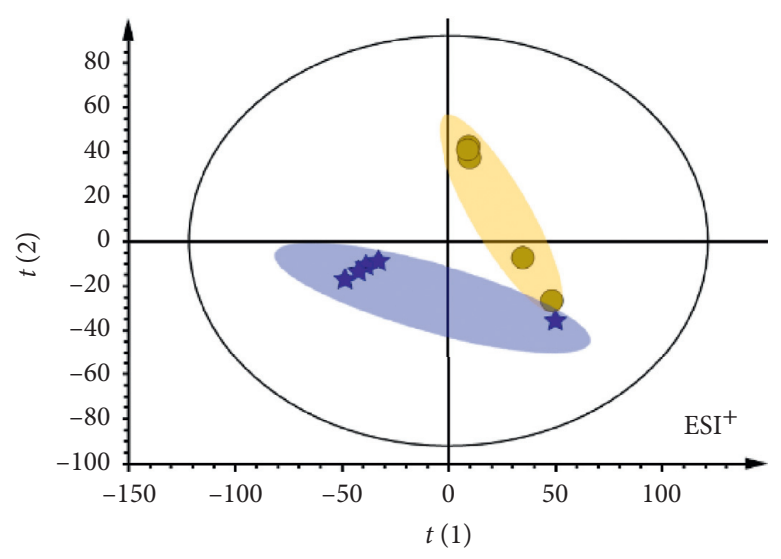

PR decoction pieces

PR formula particles

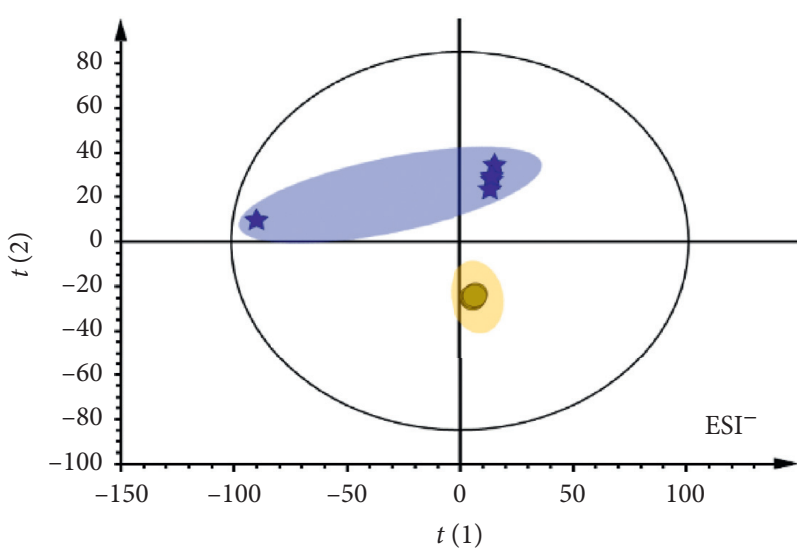

PR decoction pieces

^ PR formula particles

(a)

(b)

FIGURE 2: The scores plot obtained from PCA analysis of PR decoction pieces and formula granules. The scores plot of PCA revealed that the 10 samples were classified into two major groups in the positive mode (a) and negative mode (b).

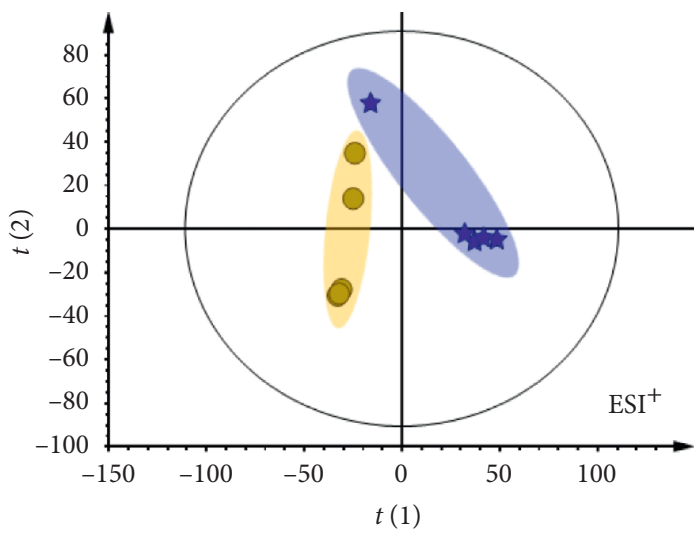

PR decoction pieces

$\downarrow$ PR formula particles

(a)

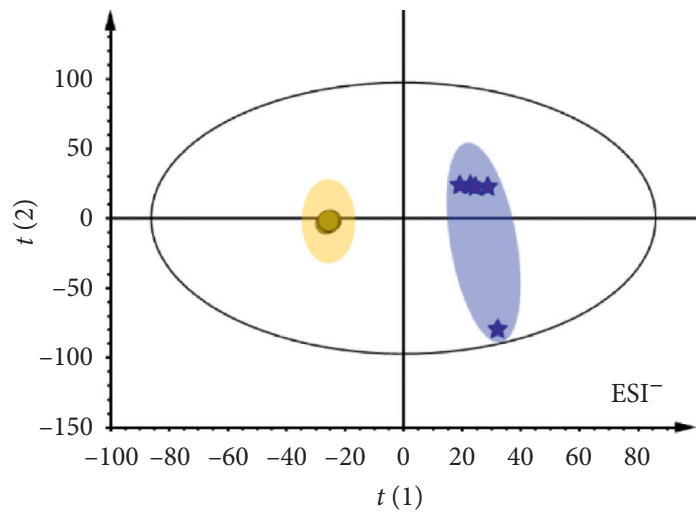

PR decoction pieces

PR formula particles

(c)

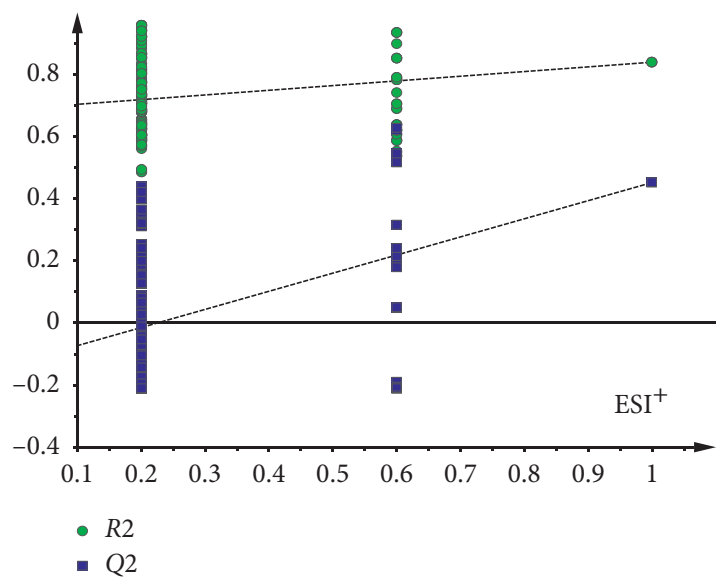

(b)

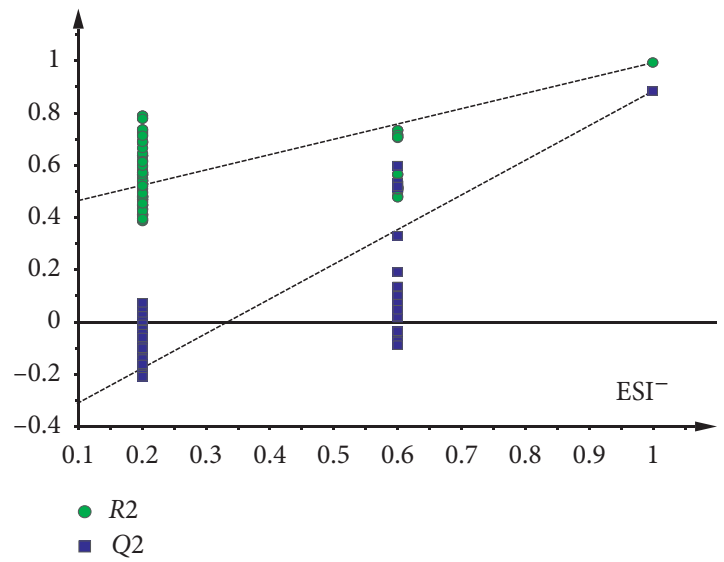

(d)

FIGURE 3: PLS-DA scores plot and the permutation test with 200 permutations of PR decoction pieces and formula granules. The scores plot of PLS-DA revealed that PR decoction pieces and PR formula granules could be clearly distinguished into two groups in the positive mode (a) and negative mode (c). The permutation test with 200 permutations indicated that PLS-DA is effective and can be used for subsequent differential component analysis in the positive mode (b) and negative mode (d). 


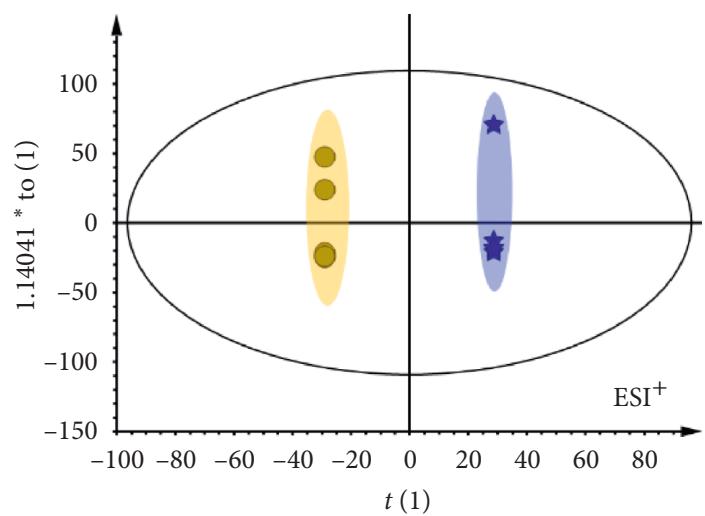

- PR decoction pieces $\star$ PR formula particles

(a)

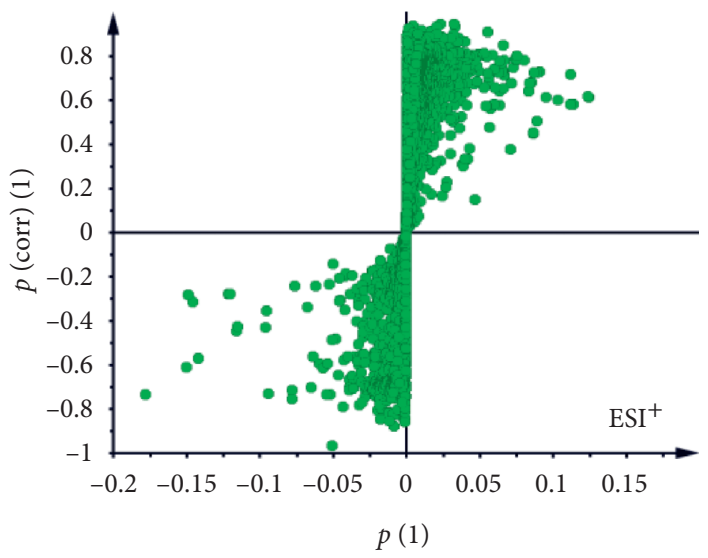

(c)

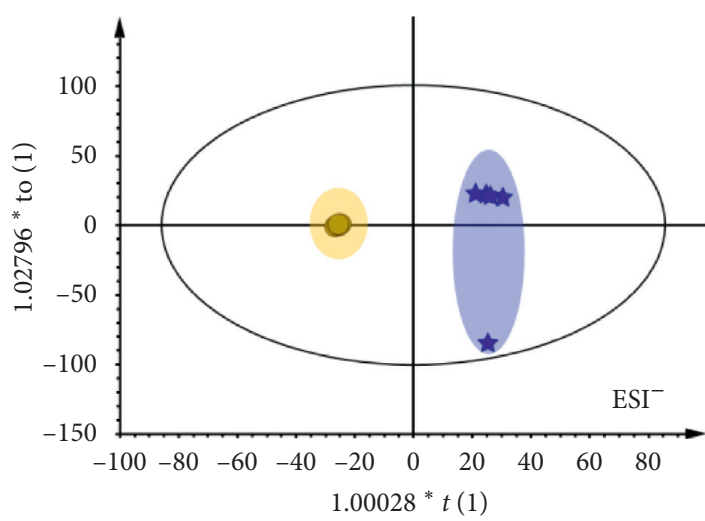

PR decoction pieces - PR formula particles

(b)

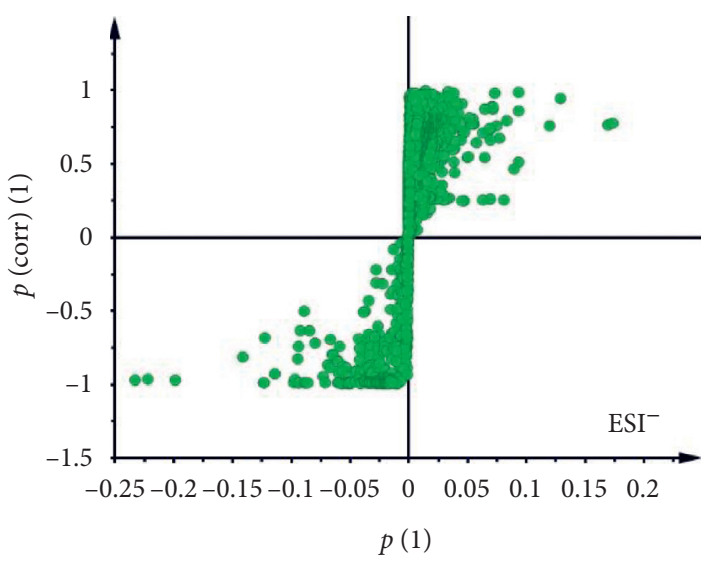

(d)

Figure 4: OPLS-DA scores plot and S-plot of PR decoction pieces and formula granules. The scores plot of OPLS-DA revealed that PR decoction pieces and PR formula granules were significantly different in the two classes in the positive mode (a) and negative mode (b). Splots indicated that the farther the distance from the central region represented the metabolites contributing more to the separation between groups in the positive mode (c) and negative mode (d).

differences in chemical constituents of PR decoction pieces (TY) and formula granules (TK). Principal component analysis (PCA) was utilized to reduce the dimensions of multivariate problems. After Vilfredo Pareto with mean centering, the data were showed as scores in a coordinate system of latent variables, which resulted from the above samples. The PCA score plot $\left(\mathrm{R}^{2} \mathrm{X}=0.805, \mathrm{Q}^{2}=0.686\right.$ in $\mathrm{ESI}^{+}$, Figure 2(a); $R^{2} X=0.886$, and $Q^{2}=0.654$ in $\mathrm{ESI}^{-}$, Figure 2(b)) showed clear separation in different dosage forms of PR in both $\mathrm{ESI}^{+}$and $\mathrm{ESI}^{-}$. This indicated that there was a significant difference in chemical constituents between TY and TK.

3.2.2. Partial Least Square Discriminant Analysis. Partial least square discriminant analysis (PLS-DA) is a supervised analysis, which extends a regression of PCA and uses class information to maximize the separation between groups of observations [23]. This frequently used classification method is categorical (categories described with dummy variables) and expressed the class membership of the statistical units.
In this research, the model parameters were $R^{2} X=0.799$, $R^{2} Y=0.840, Q^{2}=0.452$ in $\mathrm{ESI}^{+}$(Figure $3(\mathrm{a})$ ); $R^{2} X=0.884$, $R^{2} Y=0.993$, and $Q^{2}=0.885$ in $\mathrm{ESI}^{-}$(Figure $3(\mathrm{c})$ ). The PLSDA scores plot showed that TY and TK were clearly isolated into two groups, and the interclass differences were less than that in the PCA model, so the PCA model was more effective to ensure the differences in two different dosages of PR. The PLS-DA model was further validated by a permutation test with 200 permutations (Figures $3(\mathrm{~b})$ and $3(\mathrm{~d})$ ). The $R^{2}$ and $Q^{2}$ values generated by the random permutation at the left end are both smaller than the original values at the right end, indicating that the predictive power of the original model is greater than that of the random permutation $y$ variables, so the model is effective and can be used for subsequent differential component analysis [24].

\subsubsection{Orthogonal Least Squares Discriminant Analysis.} In order to further identify the significant metabolites contributing to distinction in the two forms of PR, orthogonal least squares discriminant analysis (OPLS-DA), a 


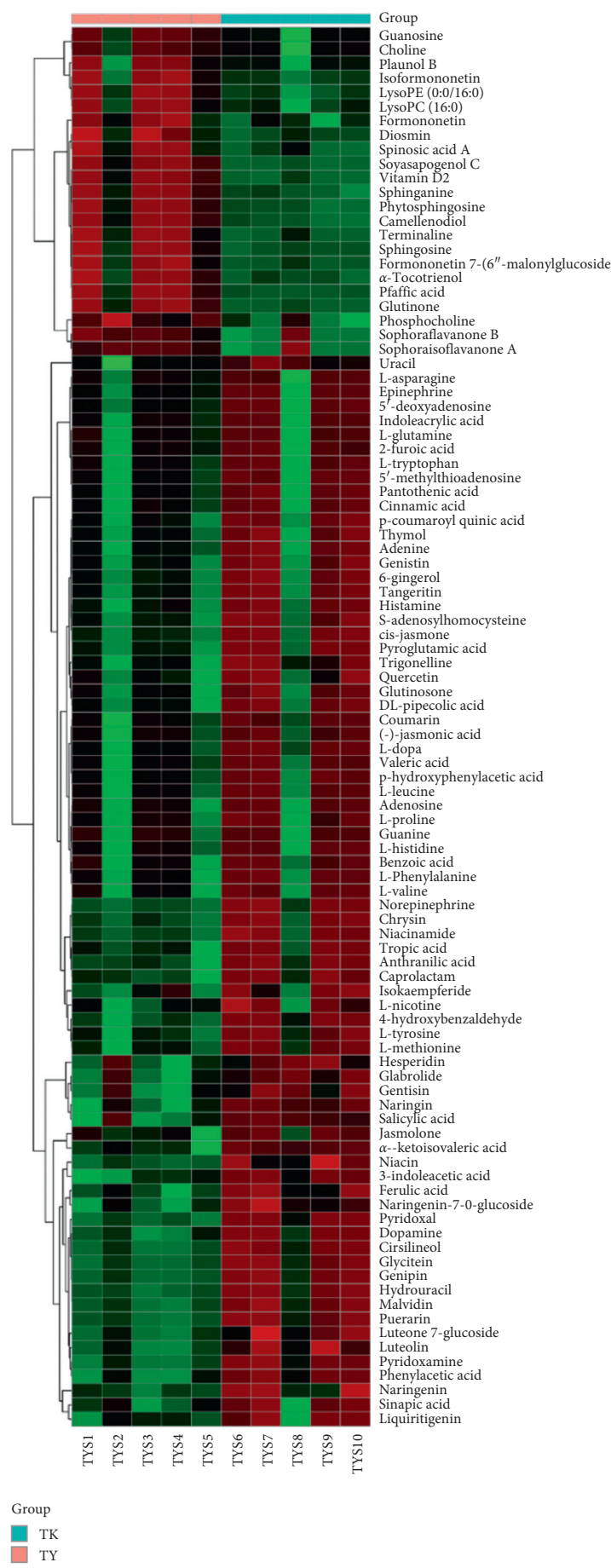

(a)
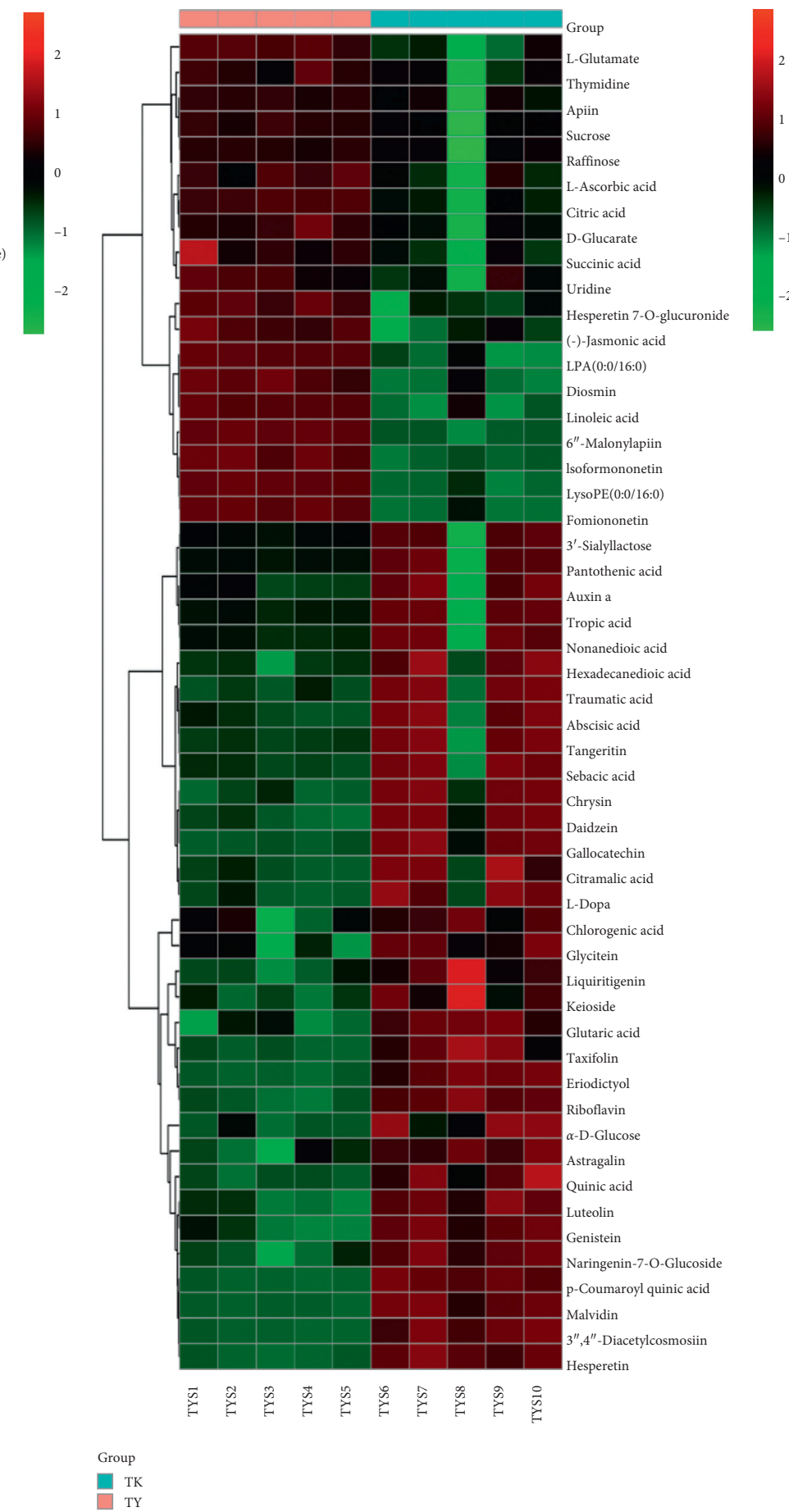

(b)

FIGURE 5: Hierarchical clustering analysis of PR decoction pieces and formula granules. Clear differentiation was observed in the heat map, colors varying from green to red graphically indicated that the relative contents of metabolites were from low to high. 98 and 52 differential chemical compositions were identified in the positive mode (a) and negative mode (b).

supervised pattern recognition approach was performed [25]. In OPLS-DA scores plot, each spot represents a sample. As shown in Figure 4, the TY group can be clearly separated from the TK group in both positive and negative modes, and the model parameters were $R^{2} X=0.933, R^{2} Y=1, Q^{2}=0.673$ in $\mathrm{ESI}^{+}$(Figure 4(a)); $R^{2} X=0.884, \quad R^{2} Y=0.993$, and
$Q^{2}=0.984$ in $\mathrm{ESI}^{-}$(Figure 4(b)), which indicated good ability of prediction and reliability of the model. To identify the metabolites contributing to the discrimination, S-plots were generated (Figure 4(c) and 4(d)). Each spot in OPLSDA scores plot and S-plots represents a variance. Farther the distance from the central region, higher is the contribution 

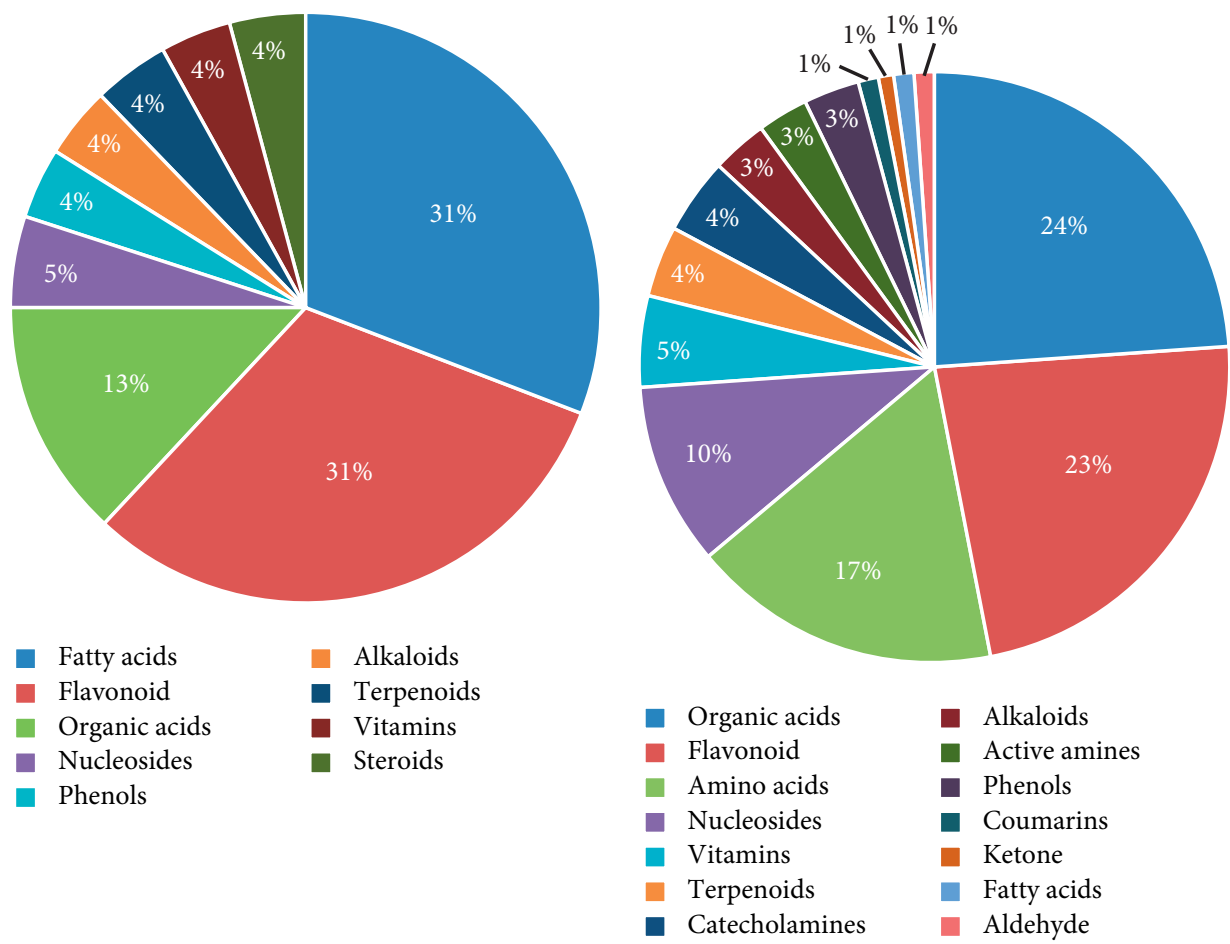

(a)
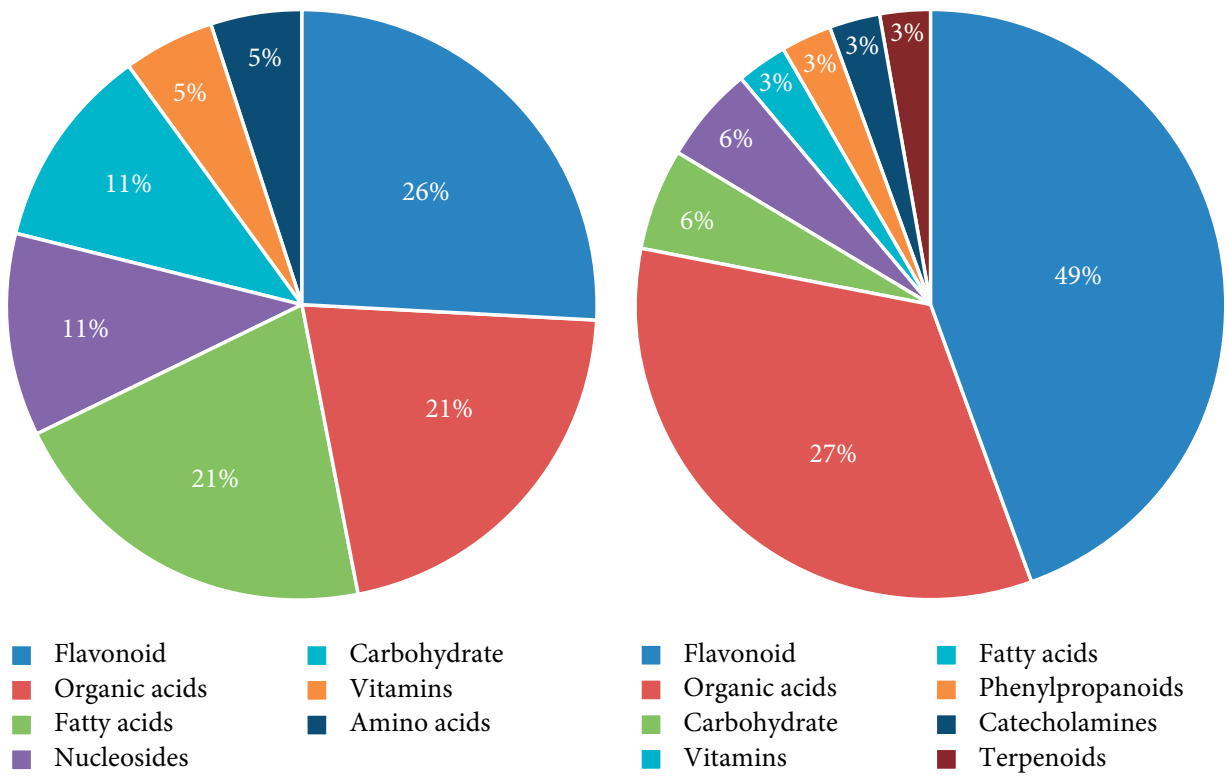

(c)

(d)

Figure 6: Comparative analysis of contents of differential chemical compositions in PR decoction pieces and formula granules. (a) The relative contents of differential chemical compositions in PR decoction pieces $>$ PR formula granules in the positive mode. (b) The relative contents of differential chemical compositions in PR decoction pieces $<$ PR formula granules in the positive mode. (c) The relative contents of differential chemical compositions in PR decoction pieces $>$ PR formula granules in the negative mode. (d) The relative contents of differential chemical compositions in $\mathrm{PR}$ decoction pieces $<\mathrm{PR}$ formula granules in the negative mode.

of the metabolites. The importance of each variance to classification was determined by the value of variable in the projection (VIP). Metabolites with the VIP value above 1.0 and $P$ value below 0.05 were considered as potential metabolic markers.
3.3. Identification of Differential Compositions with Relative Contents Analysis. VIP $>1$ of OPLS-DA combined with the $t$-test $(p<0.05)$ was used to discover the significantly differential metabolites in TY and TK. METLIN and HMDB databases are used to search the accurate mass-to-charge 
ratio to identify the differential chemical compositions. As seen from hierarchical clustering analysis (Figures 5(a) and 5(b)), a total of 98 and 52 differential chemical compositions were identified in the positive mode and negative mode, respectively, and the detailed information is given in Table S1 and Table S2.

Clear differentiation was observed in the heat map based on differential chemical compositions, and colors varying from green to red graphically indicate that the relative contents of metabolites are from low to high. In the positive mode, there were 23 differential chemical compositions that had the relative contents of TY greater than that in TK, while there were 75 differential chemical compositions that the relative contents of TY were less than that in TK. In the negative mode, there were 19 differential chemical compositions that the relative contents of TY were greater than that in TK, while there were 33 differential chemical compositions that the relative contents of TY were less than that in TK.

As shown in Figures 6(a) and 6(b), in the positive mode, the relative contents of differential chemical compositions in TY above that in TK were distributed in nine kinds of compositions. Flavonoids and fatty acids had the highest proportion, accounting for $31 \%$. The relative contents of differential chemical compositions in TY below that in TK were distributed in fourteen varieties of compositions. Among them, 18 organic acids accounted for the largest proportion (24\%), followed by flavonoids $(17,23 \%)$ and amino acids $(13,17 \%)$. In the negative mode, the relative contents of differential chemical compositions in TY above that in TK were mainly involved in seven varieties of compositions. Flavonoids $(5,26 \%)$ were the most abundant, followed by organic acids $(4,21 \%)$ and fatty acids $(4,21 \%)$ (Figure 6(c)). The relative contents of differential chemical compositions in TY below that in TK belonged to eight varieties of compositions. Among them, flavonoids accounted for the highest proportion (16, 49\%), followed by organic acids 9, 27\% (Figure 6(d)). The results showed that differential chemical compositions of PR with different dosage forms were mainly concentrated in flavonoids, organic acids, amino acids, and fatty acids. In additional, in both positive and negative modes, the amounts and types of differential chemical compositions with higher relative contents in TK were both more than those with higher relative contents in TY, for example, flavonoids with antitumor effects, including chrysin, naringin, luteolin, and genistin; antimicrobial resistance to oxidation of organic compounds, including cinnamic acid, ferulic acid, and salicylic acid; and amino acid compounds with immunepromoting effects, including L-tryptophan, L-phenylalanine, and L-tyrosine.

3.4. Metabolic Pathway Analysis. The metabolic pathway was analyzed by MetaboAnalyst website, and the Kyoto Encyclopedia of Genes and Genomes (KEGG) of the above identified compounds was introduced to MetaboAnalyst for pathway analysis. The radius of dots represents the impact value of the metabolic pathway. The larger the impact value,

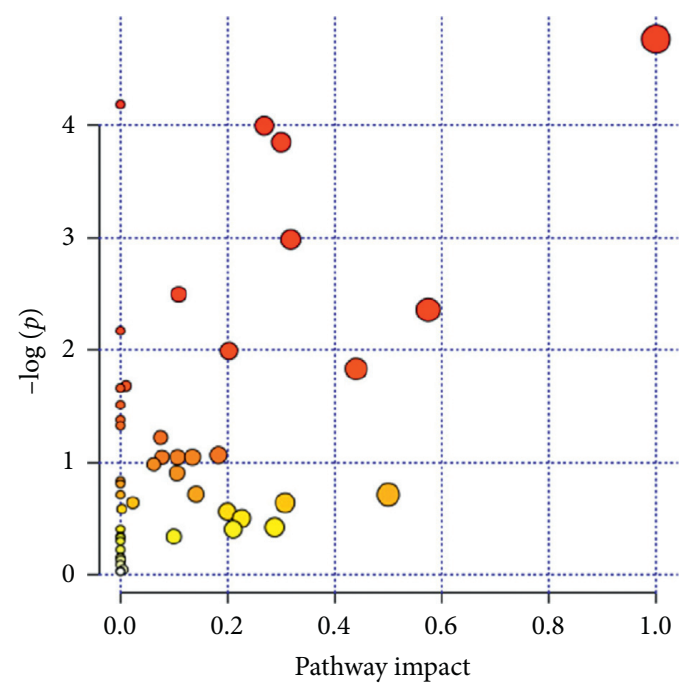

FIGURE 7: Analysis of pathway impact. Isoquinoline alkaloid biosynthesis which was the potential key metabolic pathway.

the larger the radius. The color of dots represents the $p$ value of the metabolic pathway, and the lower the $p$ value, the redder the color. The threshold value for the effect of the metabolic pathway was set to 0.6 with topological Fenix, greater than which will be selected as potential key metabolic pathways. Finally, one metabolic pathway, isoquinoline alkaloid biosynthesis (A), was coenriched (Figure 7).

\section{Conclusions}

In this research, the difference of chemical compositions in PR with different dosage forms was analyzed by HPLC-QExactive Orbitrap/MS combined with multivariate statistical analysis. Results showed that the chemical compositions exited great differences in TY and TK. A total of 98 differential chemical compositions were found in $\mathrm{ESI}^{+}$, while 52 were found in $\mathrm{ESI}^{-}$. These constituents were mainly concentrated in flavonoids, organic acids, fatty acids, and amino acids, mainly involved in the isoquinoline alkaloid biosynthesis metabolic pathways. In addition, both in positive and negative modes, the amounts and types of differential chemical compositions with higher relative contents in TK were both more than those with higher relative contents in TY, including flavonoids with the antitumor effect, organic acids containing the antibacterial antioxidant effect, and amino acids which can boost immunity.

This study will provide the basic information for exploring the influence law of different dosage forms on the synthesis and accumulation of metabolites in PR and its quality-forming mechanism. It will also provide a reliable and accurate approach for the analysis of complex samples and identification of differential chemical compositions.

\section{Data Availability}

The data used to support the findings of this study are available from the corresponding author upon request. 


\section{Conflicts of Interest}

The authors declare that they have no conflicts of interest.

\section{Authors' Contributions}

Yujiao Hua and Xiaoyuan Liu carried out the experiments, analyzed data, and composed the draft of the article. Yujiao Hua analyzed data and wrote the article. Xiaoyuan Liu provided the samples of PR. Fen Xie participated in the supervision of this study and edited the article. All authors read and approved the final draft of the article.

\section{Acknowledgments}

This work was supported by the Talent Plan of Affiliated Hospital of Jiangnan University (Wuxi No. 4 People's Hospital) (201821) and Wuxi Science and Technology Development Medical and Health Guidance Plan (NZ2019012).

\section{Supplementary Materials}

Table S1. Components identified with significant differences from Pseudostellariae Radix with different dosage forms in the positive ion mode $(p<0.05)$. Table S2. Components identified with significant differences from Pseudostellariae Radix with different dosage forms in the negative ion mode $(p<0.05)$. (Supplementary Materials)

\section{References}

[1] Chinese Pharmacopoeia Commission, Chinese Pharmacopoeia 2015. Part1, p. 68, China Medical Science Press, Beijing, China, 2015.

[2] X. S. Fu, X. H. Liu, H. Xu et al., "Research status and trends of pseudostellariae radix," Chinese Journal of New Drugs, vol. 21, no. 7, pp. 757-760, 2012.

[3] X. C. Shen, L. Tao, S. Bo et al., "Ameliorated effects of Radix Pseudostellariae on oxidative stress in rat chronic heart failure induced by acute cardiac infarction," Journal of Chinese Pharmaceutical Sciences, vol. 23, pp. 413-416, 2008.

[4] C. L. Zhang and X. X. Zhang, "Effects of polysaccharides from Pseudostellariaheterophylla on exercise endurance capacity and oxidative stress in forced swimming rats," Scientific Research and Essays, vol. 6, pp. 2360-2365, 2011.

[5] R. Sheng, X. Xu, Q. Tang et al., "Polysaccharide of radix pseudostellariae improves chronic fatigue syndrome induced by poly I:C in mice," Evidence-Based Complementary and Alternative Medicine, vol. 2011, Article ID 840516, 9 pages, 2011.

[6] S. D. Ni, L. Z. Xia, X. X. Xu et al., "Effect of pseudostellaria polysaccharides in diabetic mice by alloxan," Anhui Medical and Pharmaceutical Journal, vol. 14, pp. 521-522, 2010.

[7] W. Pang, S. Lin, Q. Dai, H. Zhang, and J. Hu, "Antitussive activity of pseudostellaria heterophylla (miq.) Pax extracts and improvement in lung function via adjustment of multi-cytokine levels," Molecules, vol. 16, no. 4, pp. 3360-3370, 2011.

[8] Y. Li, X. Shi, and Z Wu, "Near-infrared for on-line determination of quality parameter of Sophora japonica L. (formula particles): from lab investigation to pilot-scale extraction process," Pharmacognosy Magazine, vol. 11, no. 41, pp. 8-13, 2015.
[9] Y. H. Li, Z. F. Wu, Y. Y. Li, H. T. Li, and M. Yang, "Analysis on relativity of drying process of extract and pelleting quality of Chinese materia medica formula granule based on powder properties," Chinese Traditional and Herbal Drugs, vol. 48, no. 10, pp. 1930-1935, 2017.

[10] J. Han, "Comparison and analysis of free-fried Chinese herbal formulagranules and traditional Chinese herbal pieces," China Continuing Medical Education, vol. 10, no. 28, pp. 142-144, 2018.

[11] J. Chen, S. Sun, and Q. Zhou, "Direct and model-free detection of carbohydrate excipients in traditional Chinese medicine formula granules by ATR-FTIR microspectroscopic imaging," Analytical and Bioanalytical Chemistry, vol. 409, no. 11, pp. 2893-2904, 2017.

[12] N. Schauer and A. Fernie, "Plant metabolomics: towards biological function and mechanism," TRENDS in Plant Science, vol. 11, no. 10, pp. 508-516, 2006.

[13] C. Y. Li, Z. Wang, P. Wang, and X. L. Meng, “Therapeutic material basis and action mechanism of Chinese materia medicabased on metabonomic technology," Chinese Traditional and Herbal Drugs, vol. 44, no. 7, pp. 918-922, 2013.

[14] S. Lyu, S. L. Yang, Y. Rao, and Y. L. Feng, "Application of metabolomics and related technologies in research and development field of traditional Chinese medicine," China Journal of Chinese Materia Medica, vol. 43, no. 21, pp. 4182-4191, 2018.

[15] Y. J. Hua, S. N. Wang, and C Chai, "Quality evaluation of pseudostellariae radix based on simultaneous determination of multiple bioactive compontents combined with grey relational analysis," Molecules, vol. 22, no. 1, pp. 13-27, 2017.

[16] J. Tan, C. Wang, H. Zhu et al., "Comprehensive metabolomics analysis of xueshuan xinmaining tablet in blood stasis model rats using UPLC-Q/TOF-MS," Molecules, vol. 23, no. 7, pp. 1650-1665, 2018.

[17] S. Wang, Y. Hua, Y. Lin et al., "Dynamic changes of metabolite accumulation in Scrophulariae Radix based on liquid chromatography-tandem mass spectrometry combined with multivariate statistical analysis," Journal of Separation Science, vol. 40, no. 14, pp. 2883-2894, 2017.

[18] H. Zhao, Y. Yan, and C. C Wang, "Comparison of chemical constituents in magnoliae OfficinalisCortex processed by "sweating" and "non sweating" based on UltraFast liquid chromatography-triple quadrupole-time of flight mass spectrometry and gas chromatography-triple QuadrupoleMass spectrometry combined with multivariate statistical analysis," Natural Product Communications, vol. 13, no. 8, pp. 987-991, 2018.

[19] X. Zhao, Y. Zhang, X. Meng et al., "Effect of a traditional Chinese medicine preparation Xindi soft capsule on rat model of acute blood stasis: a urinary metabonomics study based on liquid chromatography-mass spectrometry," Journal of Chromatography B, vol. 873, no. 2, pp. 151-158, 2008.

[20] B. Zhuang, Z.-M. Bi, Z.-Y. Wang, L. Duan, C.-J.-S. Lai, and E.-H. Liu, "Chemical profiling and quantitation of bioactive compounds in Platycladi Cacumen by UPLC-Q-TOF-MS/MS and UPLC-DAD," Journal of Pharmaceutical and Biomedical Analysis, vol. 154, pp. 207-215, 2018.

[21] Y. Hua, Y. Hou, S. Wang et al., "Comparison of chemical compositions in pseudostellariae radix from different cultivated fields and germplasms by NMR-based metabolomics," Molecules, vol. 21, no. 11, pp. 1538-1549, 2016.

[22] Y. F. Zhou, M. Niu, and J. X Fang, "Metabonomics study on hepatoprotective of SchisandraeChinensis Fructus based on 
UPLC-Q/TOF-MS," China Journal of Chinese Materia Medica, vol. 43, no. 18, pp. 3756-3763, 2018.

[23] J. Trygg, E. Holmes, and T. Lundstedt, "Chemometrics in metabonomics," Journal of Proteome Research, vol. 6, no. 2, pp. 469-479, 2007.

[24] Z.-Y. Li, H.-J. Zhi, F.-S. Zhang et al., "Metabolomic profiling of the antitussive and expectorant plant Tussilago farfara L. by nuclear magnetic resonance spectroscopy and multivariate data analysis," Journal of Pharmaceutical and Biomedical Analysis, vol. 75, pp. 158-164, 2013.

[25] N. C. Luo, W. Ding, and J Wu, "UPLC-Q-TOF/MS coupled with multivariate statistical analysis as apowerful technique for rapidly exploring potential chemical markers to differentiate between radix paeoniae alba and radix paeoniae rubra," NaturalProduct Communications, vol. 8, no. 4, pp. 487-491, 2013. 